\title{
A Critical Review on Discussion and Traditional Teaching Methods
}

\author{
Saira $^{1^{*}}$, Nishat Zafar ${ }^{2}$, Muhammad Hafeez $^{3}$ \\ ${ }^{1 *}$ Department of Education, University of Gujrat, Gujrat, Pakistan. \\ ${ }^{2}$ Department of Education, University of Gujrat, Gujrat, Pakistan. \\ ${ }^{3}$ Research Scholar, Allama Iqbal Open University, Islamabad, Pakistan.
}

\begin{abstract}
Teaching strategy is an important part of learning. Without suitable learning method, the required goals cannot be achieved. A review study comprises of descriptive and statistical results of previous published articles on traditional lecture and discussion teaching methods has been conducted. Thirty (30) articles published from 2014-2020 have selected for the review. The review of literature indicated that form 30 selected articles, 24 articles showed statistically higher significant value for discussion teaching method and only six articles showed low significant value form discussion teaching method. So, by review it can be concluded that discussion teaching method is more effective and result oriented teaching strategy than traditional lecture method.
\end{abstract}

Keywords: Critical Thinking Skills; Cooperative; Effective; Learners Engagement; Learning Stratigies

\section{Introduction}

Education is a basic need of humanity and fundamental element for the progress of a country (Hafeez et al., 2020). The most important challenge in the educational process is the selection of suitable and effective teaching methods to make the learning process useful and to develop the critical thinking skills in the learners (Senthamarai, 2018; Tavoosy \& Jelveh, 2019). Two important factors to develop the critical thinking skills among the learners are strategy adopted by the instructor and dynamic engagement of the learners in the teaching learning process (Nelson, 2017). In the teaching-learning process, the instructor must act as a guider instead of knowledge transfer (Molbaek, 2018).

Various teaching methods like traditional lecture, discussion, project based are discussed in the literature (Farashahi \& Tajeddin, 2018; Usarov, 2019; Yusupov, 2020). The most useful teaching strategy to increase the critical thinking is the small group discussion teaching method as concluded by the various studies (Bidabadi, et al., 2016; Yli-
Panula et al., 2018; Sivarajah et al., 2019; Strubbe et al., 2020).

The traditional lecture method is a one-way conversation in which an instructor delivers the information before the audience (Gholami et al., 2016). After the lecture, the instructor gives notes and assign some tasks as homework (Gregorius, 2017). In traditional lecture teaching methods, no feedback session for the learners is conducted (Almanasef et al., 2020). Generally, very little conversation happens between the learners and instructors (Sarihan et al., 2016). The learners receive passive strategy of learning (Maqbool et al., 2018).

Another teaching method is the discussion method. The discussion teaching strategy involves the small group activities to discuss and to find the solution of the problem (Wang, 2016). The discussion teaching method is also called constructive learning process (Keshavarzi et al., 2016). The discussion teaching method is a two-way conversation method (Dewi, 2019). It is an active learning strategy (Shamsudin et al., 2017). 

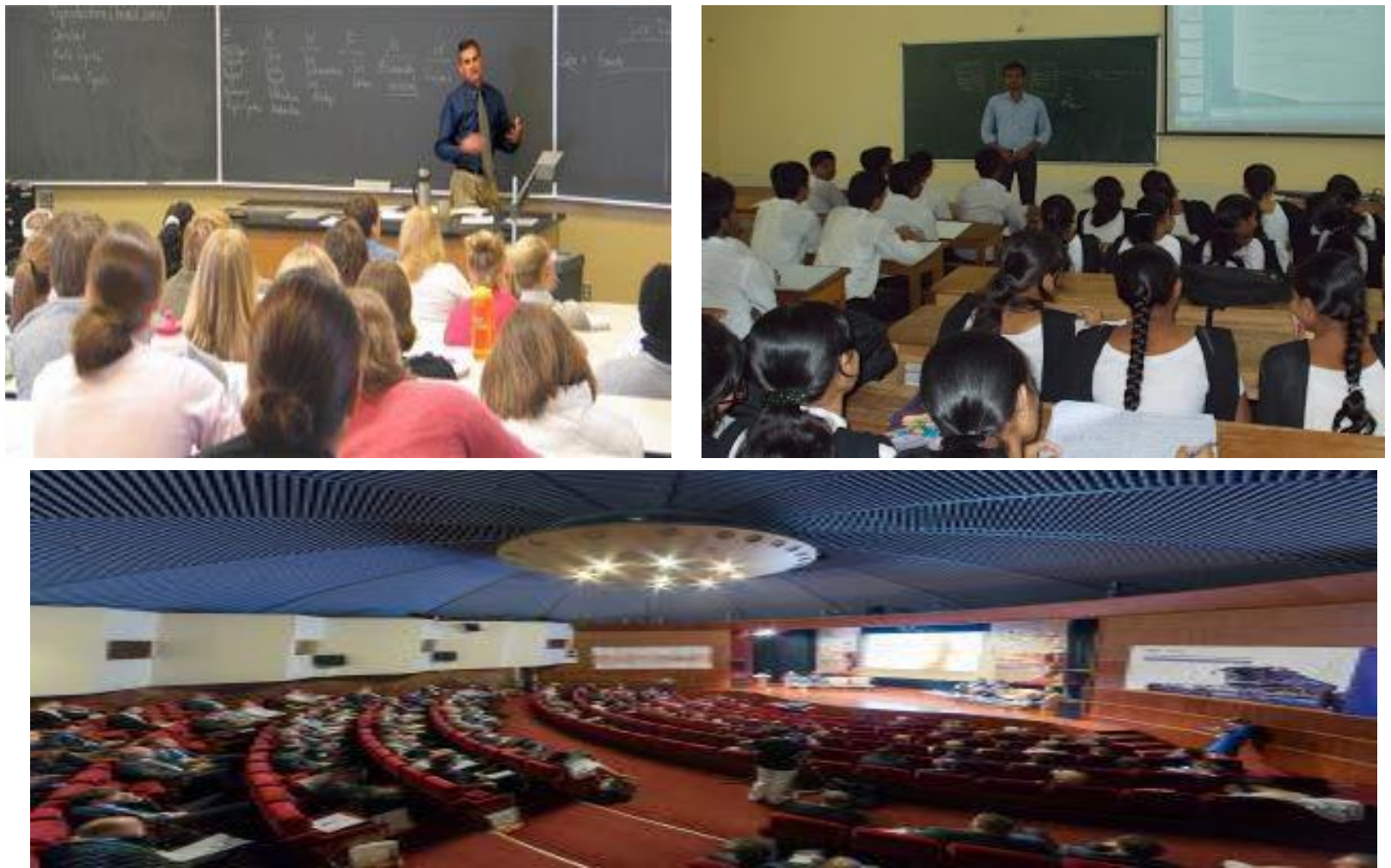

Figure.1: Some Pictorial Views of Traditional Lecture Method

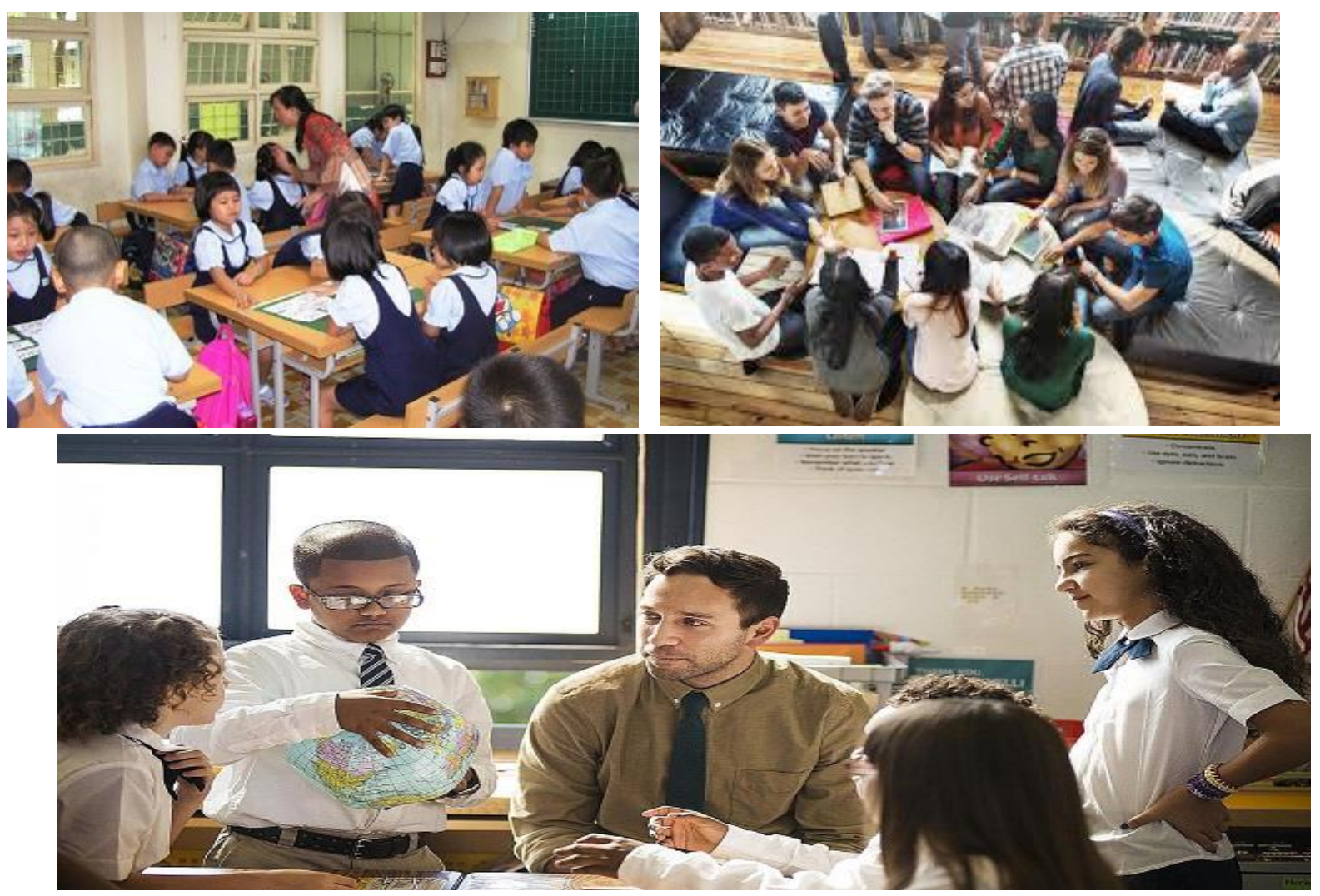

Figure.2: Some Pictorial Views of Discussion Teaching Method 
Various researches including (Dufva \& Dufva, 2016; Arise, 2018; Richards \& Graber, 2019; Bohari, 2020) concluded that traditional lecture method has abortive to transfer conceptual knowledge to the learners. So, the traditional lecture method only recommended when knowledge transfer is the main purpose (You et al.,
2017). The traditional lecture method does not develop the critical thinking skills in the learners (Carter et al., 2016; Dehghanzadeh \& Jafaraghaee, 2018). The discussion teaching method is an effective teaching strategy to develop the critical thinking skills in the learners (Fung et al., 2016; Rathakrishnan et al., 2018; Alsaleh, 2020).

\section{9 \\ Traditional Classroom \\ - Instructor prepares material to be delivered in class. \\ - Students listen to lectures and other guided instruction in class and take notes. \\ - Homework is asisgned to demonstrate understanding.}

Figure.3: Conceptual View of Traditional Lecture Method

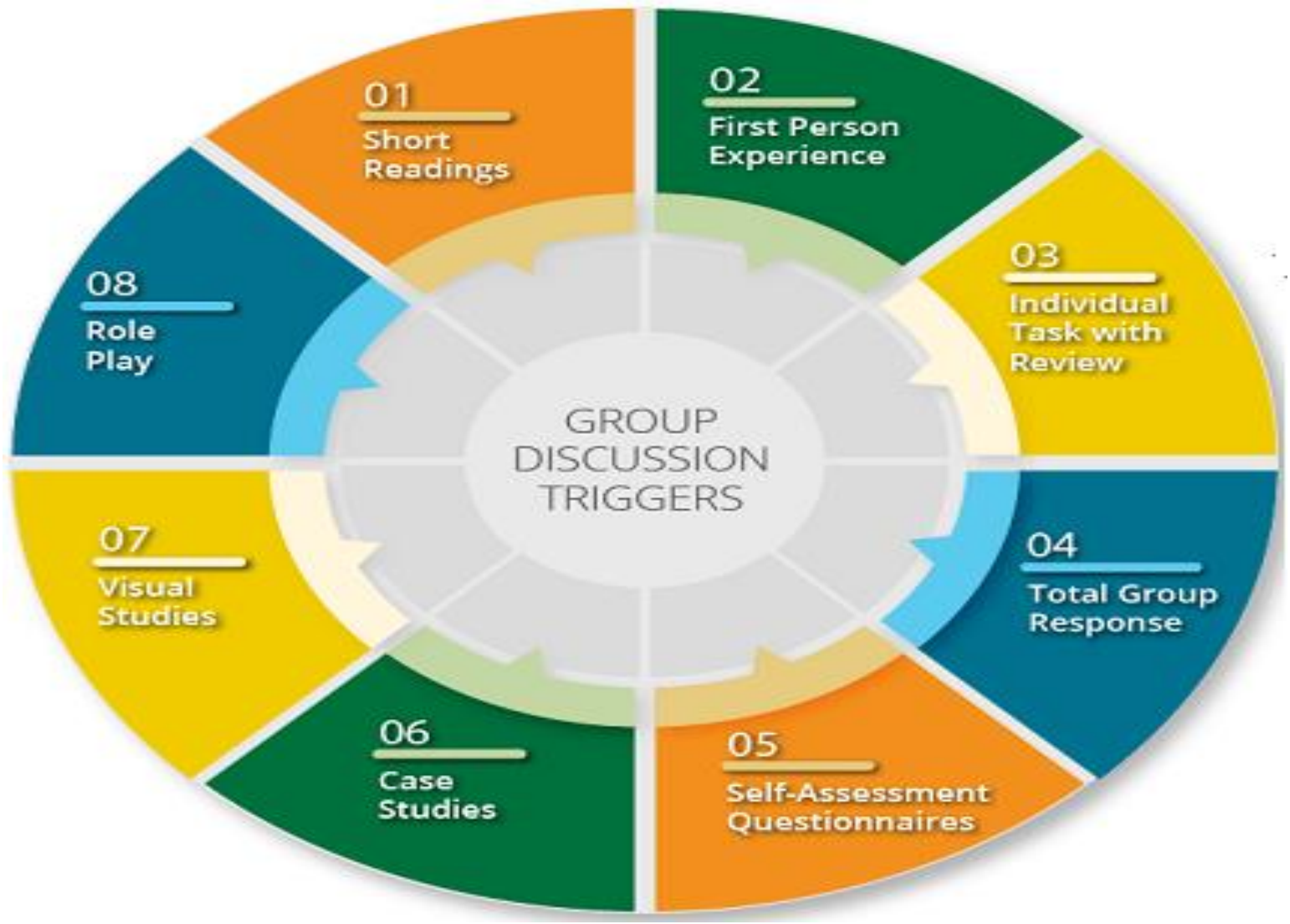

Figure.4: Conceptual view of Discussion Teaching Method 
Padugupati et al., (2017) conducted a research to find the effectiveness of various teaching methods for the development of critical thinking skills in the learners of grade 5 in General Science course. The results of the study indicated that discussion teaching method is an effective learning strategy for the development of critical thinking skills. This teaching method also improved the student's problem-solving abilities. The traditional lecture method has been found poor to develop the critical thinking skills of the learners. Rosenthal \& Walker, (2020) conducted a study to compare the teaching methods. The study concluded that discussion teaching strategy is an effective way of learning. The objective to conduct this study is to critically review the significance of discussion and traditional lecture methods statistically for an effective learning process.

\section{Review of Literature}

\section{Tradiitional Lecture Method}

It is concluded in previous researches (Lee et al., 2016; Zaidi et al., 2017; Raja, 2018;
Habibzadeh et al., 2019; Lo et al., 2020) that the learners have mixed perceptions about traditional lecture method for its effectiveness. Nurutdinova et al., (2016) conducted a study to evaluate the effectiveness of various teaching method in a primary school for grade five students. The conclusion of the study indicated that traditional lecture method has created low critical thinking skills in the learners as compared to the other teaching methods.

Some researchers (Balliu, 2017; Alaagib et al., 2019) also concluded that the traditional lecture method is a useful teaching strategy when the learning material is not available in written form like book. Zlotskaya, (2016) proposed a research to check the suitability of the teaching methods according to the learning environments. The results of the study indicated that the traditional lecture method is a useful teaching strategy especially when there are large group of learners before the instructor. The main features of traditional lecture method are shown in figure 5.

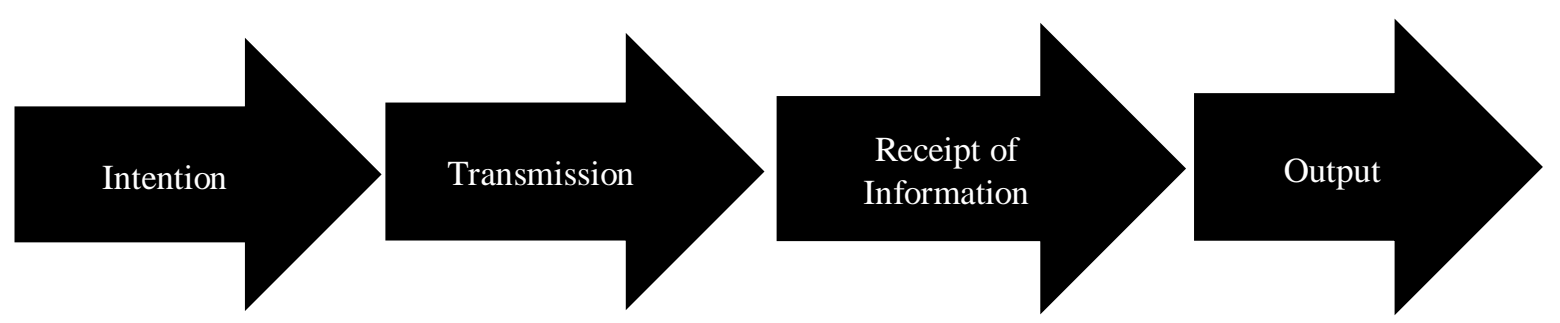

Figure.5: Main Features of Traditional Lecture Method

\section{Discussion Teaching Method}

The research of Ardeleanu, (2019) concluded that the learners gain more attention and critical thinking skills in small group discussion method. This teaching method also improves the mental abilities and level of intelligence to solve the reallife problems. The effectiveness of discussion teaching method has been proved by many researchers $(\mathrm{Ni}, 2017$; Chen et al., 2018; Chukwurah et al., 2020). Seeley, (2017) conducted a study to compare the traditional lecture and discussion teaching methods. The results of the study indicated that discussion teaching method is more effective and useful teaching-learning strategy to improve the students critical thinking and communication skills.

Nami et al., (2018) characterized that the discussion teaching method is a selfdirected and two-way conservation in which the learners discuss for the solution of the problem by sharing their ideas with each other's. The main features of discussion teaching method are shown in figure 6 


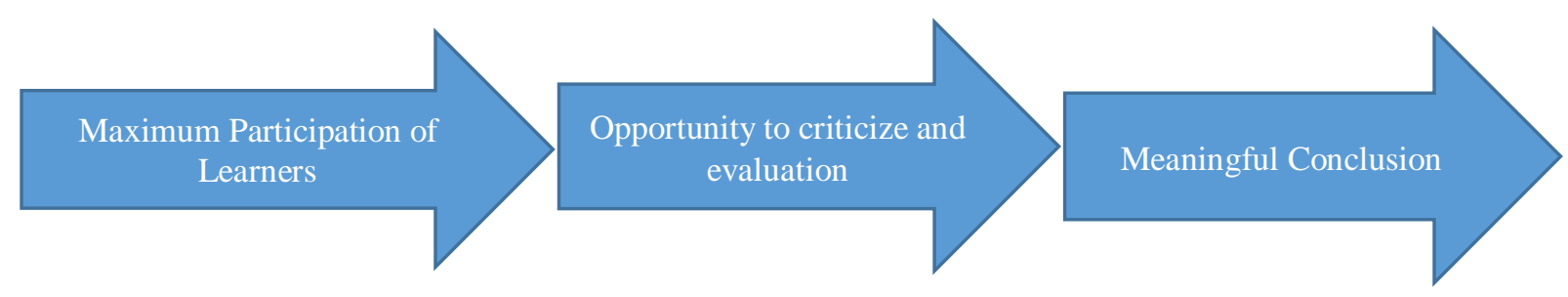

Figure.6: Main Features of Discussion Teaching Method

Many studies including (Sun \& Bin, 2018; Yi et al., 2019) recommended that new learning stratigies like small group discussion offer the learners to participate in the learning process actively. By incorporating the discussion learning strategy, the learner's abilities about critical thinking skills and cooperative learning has increased. The main septs for the preparation and conduction of discussion teaching method are shown in figure 7.

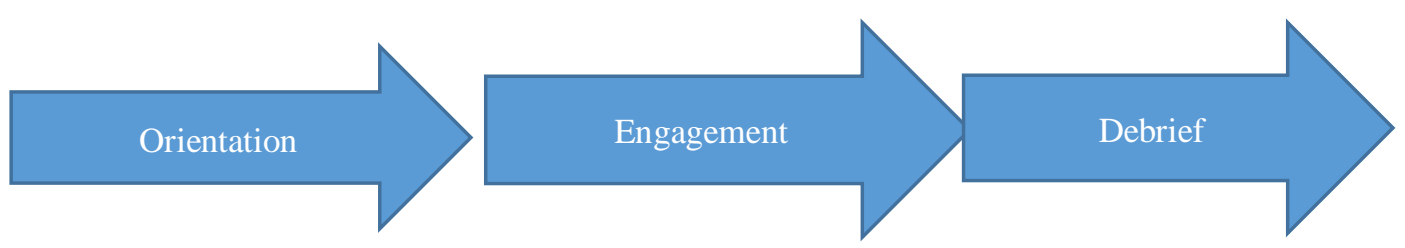

Figure.7: Steps for Preparation and Conduct of Discussion Method

The advantages and disadvantages of traditional lecture and discussion teaching methods are illustrated in table 1.

Table.1: Advantages and Disadvantages of Lecture and Discussion Teaching Methods

\begin{tabular}{|c|c|c|}
\hline $\begin{array}{l}\text { Teaching } \\
\text { Methods }\end{array}$ & Advantages & Disadvantages \\
\hline Lecture & $\begin{array}{l}\text { 1. Information can be delivered in an } \\
\text { impressive way and short time. } \\
\text { 2. It can produce interest in the subject } \\
\text { matter. } \\
\text { 3. It improves the speaking power and } \\
\text { vocabulary. } \\
\text { 4. It can be suitable for all the learners } \\
\text { by using various languages. } \\
\text { 5. The topics cab be covered by various } \\
\text { approaches. }\end{array}$ & $\begin{array}{l}\text { 1. It looks like waste of time by delivering } \\
\text { the information already presenting in } \\
\text { the books. } \\
\text { 2. The teacher has to prepare the lecture } \\
\text { by different angles. } \\
\text { 3. If the speed of instructor is fast during } \\
\text { the lecture, then it may cause problem } \\
\text { for many learners. } \\
\text { 4. The learners become passive. } \\
\text { 5. There is no interaction and cooperation } \\
\text { between the learners and the instructor. }\end{array}$ \\
\hline Discussion & $\begin{array}{l}\text { 1. Active participation of every learner } \\
\text { 2. This teaching method makes the } \\
\text { learning process interesting } \\
\text { 3. More learning than teaching } \\
\text { 4. Democrating learning } \\
\text { 5. Reflective thinking }\end{array}$ & $\begin{array}{l}\text { 1. Need more effort and time } \\
\text { 2. Solution of the problem may not be } \\
\text { found } \\
\text { 3. Lack of Guidance may occur } \\
\text { 4. Few learners may lead the discussion } \\
\text { 5. Emotional stress may be happened } \\
\text { during the discussion session }\end{array}$ \\
\hline
\end{tabular}

The conclusion of various researches about traditional lecture and discussion teaching methods is illustrated in the table 2.

Table.2: Review of Previous Researches about traditional lecture and discussion teaching methods 


\begin{tabular}{|c|c|c|c|}
\hline Reference & Class & Subject & Outcomes \\
\hline Linneman, (2019) & Undergraduate & Psychology & $\begin{array}{l}\text { Discussion teaching method significantly } \\
\text { increased the learners critical thinking } \\
\text { skills. }\end{array}$ \\
\hline Arias et al., (2016) & $\begin{array}{l}\text { First-year dental } \\
\text { students }\end{array}$ & $\begin{array}{l}\text { Endodontic rotation } \\
\text { course }\end{array}$ & $\begin{array}{l}\text { Study concluded that students scored } \\
\text { higher grades in discussion method. }\end{array}$ \\
\hline $\begin{array}{l}\text { Mutrofin et al., } \\
\text { (2019) }\end{array}$ & $\begin{array}{l}\text { theacher trainee's } \\
\text { student }\end{array}$ & Different Courses & $\begin{array}{l}\text { Discussion method significantly improved } \\
\text { the outcomes as compared to the } \\
\text { traditional lecture method. }\end{array}$ \\
\hline $\begin{array}{l}\text { Padugupati et al., } \\
\text { (2017) }\end{array}$ & $\begin{array}{l}\text { first year MBBS } \\
\text { students }\end{array}$ & Lipid chemistry & $\begin{array}{l}\text { Significantly improved the understanding } \\
\text { level of the students by discussion } \\
\text { teaching method. }\end{array}$ \\
\hline $\begin{array}{l}\text { Sudarmika et al., } \\
(2020)\end{array}$ & $\begin{array}{l}\text { Undergraduate } \\
\text { nursing student }\end{array}$ & Physical Health & $\begin{array}{l}\text { The discussion teaching method } \\
\text { significantly improves the learning } \\
\text { process. }\end{array}$ \\
\hline $\begin{array}{l}\text { Zhao \& Potter, } \\
(2016)\end{array}$ & $\begin{array}{l}\text { Third year medical } \\
\text { students }\end{array}$ & gastroschisis & $\begin{array}{l}\text { The results were significant for discussion } \\
\text { teaching method. }\end{array}$ \\
\hline Shah, (2019) & $\begin{array}{l}\text { Undergraduate } \\
\text { medical students }\end{array}$ & Surgery course & $\begin{array}{l}\text { The discussion teaching method } \\
\text { significantly improved the grades of the } \\
\text { students. }\end{array}$ \\
\hline $\begin{array}{l}\text { Ramezankhani et } \\
\text { al., } 2019\end{array}$ & Medical Students & $\begin{array}{l}\text { Blood pressure } \\
\text { related course }\end{array}$ & $\begin{array}{l}\text { Highly significant improvement was } \\
\text { found among the students studied by } \\
\text { group discussion teaching method. }\end{array}$ \\
\hline $\begin{array}{l}\text { Meguid \& Collins, } \\
\text { (2017) }\end{array}$ & $\begin{array}{l}\text { undergraduate } \\
\text { dental students }\end{array}$ & Anatomy course & $\begin{array}{l}\text { Discussion teaching method proved to be } \\
\text { very effective. }\end{array}$ \\
\hline $\begin{array}{l}\text { Azizifar \& } \\
\text { Hosseinpour, } \\
(2020)\end{array}$ & $\begin{array}{l}\text { Technical college } \\
\text { students }\end{array}$ & Pedagogy subject & $\begin{array}{l}\text { Study concluded that the discussion } \\
\text { teaching method is an effective way of } \\
\text { learning technical skills. }\end{array}$ \\
\hline Xhomara, (2020) & $\begin{array}{l}\text { Undergraduate } \\
\text { Students }\end{array}$ & Psychology & $\begin{array}{l}\text { No significant relation has been found } \\
\text { between the discussion and lecture } \\
\text { teaching methods. }\end{array}$ \\
\hline Rudolph, (2018) & $\begin{array}{l}\text { Undergraduate } \\
\text { History students }\end{array}$ & History & $\begin{array}{l}\text { Significantly improved the students } \\
\text { critical thinking skills by discussion } \\
\text { teaching method. }\end{array}$ \\
\hline Gibson, (2018) & $\begin{array}{l}\text { Undergraduate } \\
\text { nursing students }\end{array}$ & Patient Care course & $\begin{array}{l}\text { No significant difference has been found } \\
\text { between the discussion and traditional } \\
\text { lecture teachings methods. }\end{array}$ \\
\hline Safari et al., (2018) & $\begin{array}{l}\text { undergraduate } \\
\text { midwifery students }\end{array}$ & gynecology & $\begin{array}{l}\text { Discussion teaching method significantly } \\
\text { improved the learning skills of the } \\
\text { learners. }\end{array}$ \\
\hline $\begin{array}{l}\text { Shah \& Jain, } \\
(2016)\end{array}$ & $\begin{array}{l}\text { Third year MBBS } \\
\text { students }\end{array}$ & $\begin{array}{l}\text { Pharmacology } \\
\text { course }\end{array}$ & $\begin{array}{l}\text { Discussion teaching method significantly } \\
\text { improved the student's communication } \\
\text { and critical thinking skills. }\end{array}$ \\
\hline Paul et al., (2019) & $\begin{array}{l}\text { First year MBBS } \\
\text { students }\end{array}$ & Psychology course & $\begin{array}{l}\text { The discussion teaching methods } \\
\text { increased the student's academic } \\
\text { achievements and critical thinking skills. }\end{array}$ \\
\hline $\begin{array}{l}\text { Pereira \& Lima, } \\
(2018)\end{array}$ & Graduate students & Agriculture course & $\begin{array}{l}\text { No significant relation has been found } \\
\text { between the two teaching methods. }\end{array}$ \\
\hline $\begin{array}{l}\text { Mohammed \& } \\
\text { Assiri, (2020) }\end{array}$ & Bachelor students & Biochemistry & $\begin{array}{l}\text { No significant relation has been found } \\
\text { between the traditional lecture and } \\
\text { discussion teaching methods. }\end{array}$ \\
\hline $\begin{array}{l}\text { Abdulbaki et al., } \\
\text { (2018) }\end{array}$ & Master students & English & $\begin{array}{l}\text { Discussion teaching methods improved } \\
\text { the learning styles of the students in } \\
\text { comprehension. }\end{array}$ \\
\hline
\end{tabular}




\begin{tabular}{|c|c|c|c|}
\hline Amalia, (2017) & $\begin{array}{l}\text { Undergraduate } \\
\text { students }\end{array}$ & Education & $\begin{array}{l}\text { No statistical significance difference has } \\
\text { been found between traditional lecture and } \\
\text { discussion teaching methods. }\end{array}$ \\
\hline Su et al., (2016) & $\begin{array}{l}\text { Undergraduate } \\
\text { students }\end{array}$ & Clinical Medicine & $\begin{array}{l}\text { The discussion teaching method } \\
\text { significantly improved the students' } \\
\text { academic achievements. }\end{array}$ \\
\hline $\begin{array}{l}\text { Rahimimand \& } \\
\text { Abbaspour, (2016) }\end{array}$ & $\begin{array}{l}\text { students of Teacher } \\
\text { Training }\end{array}$ & $\begin{array}{l}\text { Educational } \\
\text { psychology }\end{array}$ & $\begin{array}{l}\text { Students taught by group discussion } \\
\text { method achieved better results. }\end{array}$ \\
\hline $\begin{array}{l}\text { Martikainen, } \\
(2017)\end{array}$ & College students & Art history & $\begin{array}{l}\text { Discussion teaching method increased the } \\
\text { student's academic achievements and } \\
\text { critical thinking skills. }\end{array}$ \\
\hline $\begin{array}{l}\text { Bhalli et al., } \\
(2016)\end{array}$ & 3rd year MBBS & $\begin{array}{l}\text { Basic surgery } \\
\text { course }\end{array}$ & $\begin{array}{l}\text { The discussion teaching method } \\
\text { significantly improved the students } \\
\text { leaning skills. }\end{array}$ \\
\hline $\begin{array}{l}\text { Mishra et al., } \\
(2016)\end{array}$ & $\begin{array}{l}\text { Sixth-semester } \\
\text { MBBS students }\end{array}$ & Oncology & $\begin{array}{l}\text { Discussion teaching method proved to be } \\
\text { a good instructional strategy. }\end{array}$ \\
\hline Malcom, (2018) & $\begin{array}{l}\text { Second year } \\
\text { pharmacy students }\end{array}$ & Ethics course & $\begin{array}{l}\text { Discussion teaching method created better } \\
\text { academic results. }\end{array}$ \\
\hline $\begin{array}{l}\text { Lessani et al., } \\
(2017)\end{array}$ & $\begin{array}{l}\text { Undergraduate } \\
\text { Mathematics }\end{array}$ & Geometry & $\begin{array}{l}\text { No significant improvement in the } \\
\text { academic achievements of students by } \\
\text { using discussion teaching method. }\end{array}$ \\
\hline $\begin{array}{l}\text { Rodomanchenko, } \\
\text { (2017) }\end{array}$ & $\begin{array}{l}\text { Undergraduate } \\
\text { students }\end{array}$ & English & $\begin{array}{l}\text { Students obtained better grades by } \\
\text { discussion teaching method. }\end{array}$ \\
\hline Rahmat, (2017) & $\begin{array}{l}\text { Eleventh Grade } \\
\text { Students }\end{array}$ & $\begin{array}{l}\text { English } \\
\text { Comprehension }\end{array}$ & $\begin{array}{l}\text { Comprehension skills of the learners } \\
\text { improved significantly by discussion } \\
\text { teaching method. }\end{array}$ \\
\hline Wei, (2014) & $\begin{array}{l}\text { Middle School } \\
\text { Students }\end{array}$ & General Science & $\begin{array}{l}\text { Students performed well by discussion } \\
\text { teaching strategy. }\end{array}$ \\
\hline
\end{tabular}

The review of the studies indicated that discussion method proved to be more effective and significant for the teaching-

\begin{tabular}{|c|c|c|c|c|c|}
\hline References & Method & Mean & SD & $\mathrm{p}$ & Remarks \\
\hline \multirow[t]{2}{*}{ Linneman, (2019) } & Lecture & 34.45 & 3.12 & \multirow{2}{*}{0.002} & \multirow[t]{2}{*}{ Significant } \\
\hline & Discussion & 36.32 & 2.98 & & \\
\hline \multirow[t]{2}{*}{ Arias et al., 2016} & Lecture & 3.25 & 0.98 & \multirow[t]{2}{*}{0.031} & \multirow[t]{2}{*}{ Significant } \\
\hline & Discussion & 4.43 & 0.61 & & \\
\hline \multirow[t]{2}{*}{ Mutrofin et al., 2019} & Lecture & 76.35 & 7.34 & \multirow[t]{2}{*}{0.001} & \multirow[t]{2}{*}{ Significant } \\
\hline & Discussion & 81.23 & 5.43 & & \\
\hline \multirow[t]{2}{*}{ Padugupati et al., 2017} & Lecture & 11.23 & 1.29 & \multirow[t]{2}{*}{0.047} & \multirow[t]{2}{*}{ Significant } \\
\hline & Discussion & 12.35 & 1.10 & & \\
\hline \multirow[t]{2}{*}{ Sudarmika et al., 2020} & Lecture & 46.32 & 2.34 & \multirow[t]{2}{*}{0.0001} & \multirow[t]{2}{*}{ Significant } \\
\hline & Discussion & 40.91 & 2.98 & & \\
\hline \multirow[t]{2}{*}{ Zhao \& Potter, (2016) } & Lecture & 17.34 & 1.79 & \multirow[t]{2}{*}{0.002} & \multirow[t]{2}{*}{ Significant } \\
\hline & Discussion & 21.83 & 1.33 & & \\
\hline \multirow[t]{2}{*}{ Shah, (2019) } & Lecture & 6.09 & 0.735 & \multirow[t]{2}{*}{0.0013} & \multirow[t]{2}{*}{ Significant } \\
\hline & Discussion & 9.68 & 0.453 & & \\
\hline \multirow[t]{3}{*}{ Ramezankhani et al., 2019} & Lecture & 11.42 & 1.39 & \multirow[t]{3}{*}{0.001} & \multirow[t]{3}{*}{ Significant } \\
\hline & Discussion & 16.31 & 0.92 & & \\
\hline & Discussion & 3.27 & 0.33 & & \\
\hline
\end{tabular}

learning process. The statistical results of these studies are illustrated in table 3 . 


\begin{tabular}{|c|c|c|c|c|c|}
\hline \multirow[t]{2}{*}{ Meguid \& Collins, (2017) } & Lecture & 34.56 & 5.34 & \multirow[t]{2}{*}{0.0011} & \multirow[t]{2}{*}{ Significant } \\
\hline & Discussion & 38.68 & 4.67 & & \\
\hline \multirow{2}{*}{$\begin{array}{l}\text { Azizifar \& Hosseinpour, } \\
(2020)\end{array}$} & Lecture & 6.59 & 1.51 & \multirow[t]{2}{*}{0.006} & \multirow[t]{2}{*}{ Significant } \\
\hline & Discussion & 7.29 & 1.23 & & \\
\hline \multirow[t]{2}{*}{ Xhomara, (2020) } & Lecture & 5.78 & 0.91 & \multirow[t]{2}{*}{0.09} & \multirow[t]{2}{*}{ Non-Significant } \\
\hline & Discussion & 4.36 & 1.10 & & \\
\hline \multirow[t]{2}{*}{ Rudolph, (2018) } & Lecture & 3.25 & 0.39 & \multirow[t]{2}{*}{0.0001} & \multirow[t]{2}{*}{ Significant } \\
\hline & Discussion & 5.42 & 0.19 & & \\
\hline \multirow[t]{2}{*}{ Gibson, (2018) } & Lecture & 4.32 & 0.61 & \multirow[t]{2}{*}{0.0079} & \multirow[t]{2}{*}{ Non-Significant } \\
\hline & Discussion & 3.76 & 0.76 & & \\
\hline \multirow[t]{2}{*}{ Safari et al., (2018) } & Lecture & 7.35 & 1.76 & \multirow[t]{2}{*}{0.0002} & \multirow[t]{2}{*}{ Significant } \\
\hline & Discussion & 9.32 & 1.23 & & \\
\hline \multirow[t]{2}{*}{ Shah \& Jain, (2016) } & Lecture & 17.65 & 3.13 & \multirow[t]{2}{*}{0.0003} & \multirow[t]{2}{*}{ Significant } \\
\hline & Discussion & 19.25 & 2.98 & & \\
\hline \multirow[t]{2}{*}{ Paul et al., (2019) } & Lecture & 78.34 & 11.34 & \multirow[t]{2}{*}{0.00001} & \multirow[t]{2}{*}{ Significant } \\
\hline & Discussion & 85.86 & 10.79 & & \\
\hline \multirow[t]{2}{*}{ Pereira \& Lima, (2018) } & Lecture & 17.61 & 2.45 & \multirow[t]{2}{*}{0.071} & \multirow[t]{2}{*}{ Non-Significant } \\
\hline & Discussion & 16.21 & 2.91 & & \\
\hline \multirow{2}{*}{$\begin{array}{l}\text { Mohammed \& Assiri, } \\
\text { (2020) }\end{array}$} & Lecture & 7.21 & 3.23 & 0.081 & Non-Significant \\
\hline & Discussion & 6.32 & 3.54 & & \\
\hline Abdulbaki et al., (2018) & Lecture & 2.36 & 0.91 & 0.0026 & Significant \\
\hline & Discussion & 3.24 & 0.63 & & \\
\hline Amalia, (2017) & Lecture & 7.23 & 0.82 & 0.078 & Non-Significant \\
\hline & Discussion & 6.71 & 0.97 & & \\
\hline Su et al., (2016) & Lecture & 3.21 & 0.89 & 0.021 & Significant \\
\hline & Discussion & 4.35 & 0.71 & & \\
\hline Rahimimand \& Abbaspour, & Lecture & 9.27 & 2.39 & 0.031 & Significant \\
\hline & Discussion & 10.11 & 2.09 & & \\
\hline Martikainen, (2017) & Lecture & 17.16 & 2.89 & 0.0003 & Significant \\
\hline & Discussion & 18.73 & 2.13 & & \\
\hline Bhalli et al., (2016) & Lecture & 15.67 & 3.19 & 0.009 & Significant \\
\hline & Discussion & 18.90 & 2.79 & & \\
\hline Mishra et al., (2016) & Lecture & 74.25 & 8.73 & 0.009 & significant \\
\hline & Discussion & 77.25 & 7.98 & & \\
\hline Malcom, (2018) & Lecture & 26.57 & 4.65 & 0.009 & Significant \\
\hline & Discussion & 28.31 & 3.89 & & \\
\hline
\end{tabular}




\begin{tabular}{llllll}
\hline Lessani et al., (2017) & Lecture & 16.78 & 1.20 & \multirow{2}{*}{0.812} & Non-Significant \\
\cline { 2 - 4 } & Discussion & 15.40 & 1.45 & & \\
\hline Rodomanchenko, (2017) & Lecture & 36.50 & 2.34 & \multirow{2}{*}{0.0005} & Significant \\
\cline { 2 - 4 } & Discussion & 37.45 & 2.03 & \\
\hline Rahmat, (2017) & Lecture & 5.34 & 1.09 & 0.004 & Significant \\
\cline { 2 - 4 } & Discussion & 7.20 & 0.72 & \\
\hline Wei, (2014) & Lecture & 2.34 & 0.65 & \multirow{2}{*}{0.0002} & Significant \\
\cline { 2 - 4 } & Discussion & 2.79 & 0.43 & & \\
\hline
\end{tabular}

The statistical results of the previous studies indicated that discussion teaching method is more significant as compared to the traditional lecture method. The students learned more actively and dynamically by securing more academic grades in the discussion teaching method.

Discussion

Various learning stratigies are available for the learners according to the learning environments and situations but traditional lecture strategy is the most applicable learning strategy around the globe. The traditional lecture method is a simple method in which an instructor delivers the information before a large group of audience. There is no feedback session in the traditional lecture method. The traditional lecture method lacks innovation, creativity, critical thinking skills and other important learning abilities in the learners (Allen et al., 2016). Many instructors and researchers (Osmani et al., 2018; Habibzadeh et al., 2019) concluded that traditional lecture method is not suitable for learner's mental ability. In every part of the world, speakers and learners of English can be seen(Wang, Akhter, \& Qureshi, 2020)

On the other hand, discussion teaching method has been proved to be very effective and constructive teaching method by many instructors and researchers (Eltanskaya et al., 2017; Gal et al., 2018). This teaching method improves the learner's critical and problem solution ability. In the present study a descriptive and statistical review has been conducted to the highlight the differences and significant of traditional lecture and discussion teaching methods. The review showed that discussion teaching method is more effective and useful teaching method than traditional lecture teaching method.

\section{Conclusion}

A review study has been conducted to indicate the importance of traditional lecture and discussion teaching methods. The descriptive and statistical results of 30 studies published from 2014-2020 have been selected for review. The review of literature indicated that 24 studies has more significant value for discussion teaching method and only 6 studies have no significant value for discussion teaching method. So, it is concluded from the review that discussion teaching method is more effective method than traditional lecture method.

\section{Conflict of Interest}

No conflict of interest has been found between the authors.

References

1. Abdulbaki, K., Suhaimi, M., Alsaqqaf, A., \& Jawad, W. (2018). The Use of the Discussion Method at University: Enhancement of Teaching and Learning. International Journal of Higher Education, 7(6), 118-128.

2. Alaagib, N. A., Musa, O. A., \& Saeed, A. M. (2019). Comparison of the effectiveness of lectures based on problems and traditional lectures in physiology teaching in 
Sudan. $B M C$

medical

education, 19(1), 365.

3. Allen, P., Withey, P., Lawton, D., \& Aquino, C. T. (2016). Andragogical Teaching Methods to Enhance Non-Traditional Student Classroom Engagement. Journal of Educational Technology, 13(2), 4759.

4. Almanasef, M., Almaghaslah, D., Portlock, J., \&Chater, A. (2020). Qualitative investigation of the flipped classroom teaching approach as an alternative to the traditional lecture. Pharmacy Education, 20, p142-150.

5. Alsaleh, N. J. (2020). Teaching Critical Thinking Skills: Literature Review. Turkish Online Journal of Educational TechnologyTOJET, 19(1), 21-39.

6. Amalia, R. (2017). Improving listening and speaking skills by using animation videos and discussion method. Pedagogy: Journal of English Language Teaching, 2(1), 30-36.

7. Ardeleanu, R. (2019). TRADITIONAL AND MODERN TEACHING METHODS IN MATHEMATICS. Journal of Innovation in Psychology, Education and Didactics, 23(2), 133-140.

8. Arias, A., Scott, R., Peters, O. A., McClain, E., \& Gluskin, A. H. (2016). Educational outcomes of small-group discussion versus traditional lecture format in dental students' learning and skills acquisition. Journal of Dental Education, 80(4), 459-465.

9. Arise, N. (2018). The Effectiveness of Small Group Discussion Method in The Teaching of Reading

\section{Comprehension (Doctoral} dissertation, Thesis).

10. Azizifar, S. N., \& Hosseinpour, M. (2020). The Effect of Group Discussion and Lecture Teaching Methods on Students' Academic Performance in Pedagogical Curriculum. Educational Development of Judishapur, 11(Supplement), 1-10.

11. Balliu, V. (2017). Modern Teaching Versus Traditional Teaching-Albanian Teachers Between Challenges and Choices. European Journal of Multidisciplinary Studies, 2(4), 2026.

12. Bhalli, M. A., Sattar, A., \& Asif, M. (2016). Teaching strategies. The Professional Medical Journal, 23(05), 614-619.

13. Bidabadi, N. S., ISFAHANI, A. N., Rouhollahi, A., \& Khalili, R. (2016). Effective teaching methods in higher education: requirements and barriers. Journal of advances in medical education \& professionalism, 4(4), 170.

14. Bohari, L. (2020). Improving speaking skillsthrough small group discussion at eleventh grade students of SMA Plus Munirul Arifin NW Praya. JOLLT Journal of Languages and Language Teaching, 7(1), 68-81.

15. Carter, A. G., Creedy, D. K., \& Sidebotham, M. (2016). Efficacy of teaching methods used to develop critical thinking in nursing and midwifery undergraduate students: A systematic review of the literature. Nurse education today, 40, 209-218.

16. Chen, C. C., Wu, M. C., \& Wu, T. T. (2018, August). Discussion on the Teaching and Learning 
Innovation of Higher-Order

Thinking. In International

Conference on Innovative

Technologies and Learning (pp. 609-615). Springer, Cham.

17. Chukwurah, L. N., Abbah, O. I., Iweama, C. N.,Ogugua, J. E., \& Ameh, J. (2020). Students' achievement in physical and health education: Effect of discussion teaching method. Int. J. Hum. Mov. Sport. Sci, 8(3), 86-90.

18. Dehghanzadeh, S., \& Jafaraghaee, F. (2018). Comparing the effects of traditional lecture and flipped classroom on nursing students' critical thinking disposition: A quasi-experimental study. Nurse Education Today, 71, 151-156.

19. Dewi, P. (2019). Group discussion and ICTs inteaching and learning English in large classes. Inovish Journal, 4(2), 20-32.

20. Dufva, T., \& Dufva, M. (2016). Metaphors of code-Structuring and broadening the discussion on teaching children to code. Thinking Skills and Creativity, 22, 97-110.

21. Eltanskaya, E., Linkova, Y., Popova, O., Arzhanovskaya, A., \& Kulichenko, Y. (2017, November). Types of interactive methods in teaching English to students of economics. In 7th International Scientific and Practical Conference" Current issues of linguistics and didactics: The interdisciplinary approach in humanities"(CILDIAH 2017). Atlantis Press.

22. Farashahi, M., \& Tajeddin, M. (2018). Effectiveness of teaching methods in business education: A comparison study on the learning outcomes of lectures, case studies and simulations. The International
Journal of Management

Education, 16(1), 131-142.

23. Fung, D. C. L., To, H., \& Leung, K. (2016). The influence of collaborative group work on students' development of critical thinking: The teacher's role in facilitating group discussions. Pedagogies: $\quad A n$ International Journal, 11(2), 146166.

24. Gal, B., Rubio, M., Iglesias, E., \& González, P. (2018). Evaluation of participatory teaching methods in undergraduate medical students' learning along the first academic courses. PloS one, 13(1), e0190173.

25. Gholami, M., Moghadam, P. K., Mohammadipoor, F., Tarahi, M. J., Sak, M., Toulabi, T., \& Pour, A. H. H. (2016). Comparing the effects of problem-based learning and the traditional lecture method on critical thinking skills and metacognitive awareness in nursing students in a critical care nursing course. Nurse education today, 45 , 16-21.

26. Gibson, S. L. (2018). Bringing more critical thinking into lectures and discussions.

27. Gregorius, R. M. (2017). Performance of underprepared students in traditional versus animation-based flipped-classroom settings. Chemistry Education Research and Practice, 18(4), 841848.

28. Habibzadeh, H., Rahmani, A., Rahimi, B., Rezai, S. A., Aghakhani, N., \& Hosseinzadegan, F. (2019). Comparative study of virtual and traditional teaching methods on the interpretation of cardiac dysrhythmia in nursing 
students. Journal of Education and Health Promotion, 8.

29. Habibzadeh, H., Rahmani, A., Rahimi, B., Rezai, S. A., Aghakhani, N., \& Hosseinzadegan, F. (2019). Comparative study of virtual and traditional teaching methods on the interpretation of cardiac dysrhythmia in nursing students. Journal of Education and Health Promotion, 8.

30. Hafeez, M., Kazmi, Q. A., Tahira, F., Hussain, M. Z., Ahmad, S., Yasmeen, A., ... \& Saqi, M. I. (2020). Impact of School Enrolment Size on Student's Achievements. Indonesian Journal of Basic Education, 3(1), 17-21. https://www.stkiprokania.ac.id/ejurnal/index.php/IJOBE/article/vie $\underline{\mathrm{w} / 302}$

31. Keshavarzi, Z., Akbari, H., Forouzanian, S., \& Sharifian, E. (2016). Comparison the Students Satisfaction of Traditional and Integrated Teaching Method in Physiology Course. Education Strategies in Medical Sciences, 8(6), 21-27.

32. Lee, J., Lee, Y., Gong, S., Bae, J., \& Choi, M. (2016). A metaanalysis of the effects of nontraditional teaching methods on the critical thinking abilities of nursing students. BMC medical education, 16(1), 240.

33. Lessani, A., Yun, A. S. M., \& Bakar, K. B. A.(2017). Comparison of new mathematics teaching methods with traditional method. People: International Journal of Social Sciences, 3(2).

34. Linneman, J. A. (2019). Share, show, and tell: Group discussion or simulations versus lecture teaching strategies in a research methods course. Teaching Sociology, 47(1), 22-31.

35. Lo, S., Abaker, A. S. S., Quondamatteo, F., Clancy, J., Rea, P., Marriott, M., \& Chapman, P. (2020). Use of a virtual 3D anterolateral thigh model in medical education: Augmentation and not replacement of traditional teaching?. Journal of Plastic, Reconstructive \& Aesthetic Surgery, 73(2), 269-275.

36. Malcom, D. R. (2018). Teaching and assessing clinical ethics through group reading experience and student-led discussion. Currents in Pharmacy Teaching and Learning, 10(5), 602-610.

37. Martikainen, J. M. (2017). Making pictures, writing about pictures, discussing pictures and lecturediscussion as teaching methods in art history. Art History Pedagogy \& Practice, 2(1), 4.

38. Meguid, E. A., \& Collins, M. (2017). Students' perceptions of lecturing approaches: traditional versus interactive teaching. Advances in medical education and practice, 8, 229.

39. Mishra, A. K., Manikandan, M., Kumar, R., Chauhan, R. C., Purty, A. J., Bazroy, J., \& Singh, Z. (2016). Concomitant use of handouts, group and panel discussion as a teaching technique for undergraduate medical students. Int J Innov Med Educ Res, 2, 18-22.

40. Mohammed, M., \& Assiri, M. A. (2020). Effectiveness of structured handouts lectures and oral discussion on the performance of science students in the basic biochemistry course. 
41. Molbaek, M. (2018). Inclusive teaching strategies-dimensions and agendas. International Journal of Inclusive Education, 22(10), 10481061.

42. Mutrofin, M., Degeng, I., Ardhana, I. W., \& Setyosari, P. (2019). The Effect of Instructional Methods (Lecture-Discussion versus Group Discussion) and Teaching Talent on Teacher Trainees Student Learning Outcomes.

43. Nami, F., Marandi, S. S., \& Sotoudehnama, E. (2018). Interaction in a discussion list: An exploration of cognitive, social, and teaching presence in teachers' online collaborations. ReCALL: the Journal of EUROCALL, 30(3), 375-398.

44. Nelson, A.E., 2017. Methods faculty use to facilitate nursing students' critical thinking. Teach. Learn. Nurs. 12 (1), 6266.https://doi.org/10.1016/j.teln.20 16.09.007.

45. Ni, Z. (2017, December). Discussion on Case Teaching Method Based on Computational Thinking in Programming Teaching. In 2017 International Conference on Social science, Education and Humanities Research (ICSEHR 2017). Atlantis Press.

46. Nurutdinova, A. R., Perchatkina, V. G., Zinatullina, L. M., Zubkova, G. I., \& Galeeva, F. T. (2016). Innovative teaching practice: Traditional and alternative methods (challenges and implications). International journal of environmental and science education, 11(10), 38073819.
47. Osmani, M., Hindi, N. M., \& Weerakkody, V. (2018). Developing employability skills in information system graduates: Traditional vs. innovative teaching methods. International Journal of Information and Communication Technology Education (IJICTE), 14(2), 17-29.

48. Padugupati, S., Joshi, K. P., Yamini, D., Chary, R.S., \& Sarma, D. V. H. S. (2017). Educational outcomes of small group discussion versus traditional lecture among first year undergraduate medical students. Journal of Education Technology in Health Sciences, 4(3), 93-96.

49. Paul, P., Subramanyam, L., Raghunathan, G., \& Arumugam, B. (2019). Learner Perceptions Regarding the Lecture and the Small Group Discussion as Teaching/Learning Methods in Physiology". Bangladesh Journal of Medical Science, 18(2), 274278.

50. Pereira, W. O., \& Lima, F. T. (2018). Challenge, Discussion and Conclusion: an active teaching strategy to turn traditional lectures into collaborative classes. Einstein (São Paulo), 16.

51. Rahimimand, M., \& Abbaspour, A. (2016). The Relationship between teaching methods (group discussion, questions and answers, scientific demonstrationand lectures) with Student achievement motivation. Educational

Psychology, 12(39), 1-24.

52. Rahmat, A. (2017). Small Group Discussion strategy towards students' reading comprehension of SMA Negeri 11 Bulukumba. Metathesis: Journal of 
English Language, Literature, and Teaching, 1(2).

53. Raja, F. U. (2018). Comparing Traditional Teaching Method and Experiential Teaching Method Using Experimental Research. Journal of Education and Educational Development, 5(2), 276-288.

54. Ramezankhani, A., Pooresmaeil, A., Rakhshandehrou, S., \& Khodakarim, S. (2019). Comparing the effects of group discussion and lecture educational methods on preventive behaviors of high blood pressure in Eslamshahr women. Researcher Bulletin of Medical Sciences, 1(24), e19-e19.

55. Rathakrishnan, M., Ahmad, R., \& Choi, L. J. (2018). Padlet Online Discussion in Enhancing Students' macro Critical Thinking Skills. Int. J. Latest Res. Humanit. Soc. Sci. IJLRHSS, 1(03), 121-126.

56. Richards, K. A. R., \& Graber, K. C. (2019). Retention in PETE: Survey results and discussion. Journal of Teaching in Physical Education, 38(1), 53-60.

57. Rodomanchenko, A. (2017). Roundtable Discussion in Language Teaching: Assessing Subject Knowledge and Language Skills. Journal of Language and Education, 3(4), 44-51.

58. Rosenthal, S., \& Walker, Z. (2020). ExperiencingLive Composite Video Lectures: Comparisons with Traditional Lectures and Common Video Lecture Methods. International Journal for the Scholarship of Teaching and Learning, 14(1), 8.

59. Rudolph, R. M. (2018). to lecture or discuss? teaching non-native english speakers studying international relations at webster university, thailand. In Proceedings of the International Conference on Education (Vol. 4, No. 1, pp. 112-125).

60. Safari, M., Yazdanpah, B., Mahmoudi, F., \& Yaghobean, N. (2018). Comparing students' rate of learning through lecturing and peer group teaching and study their viewpoints. Research in Medical Education, 10(3), 24-34.

61. Sarıhan, A., Oray, N. C., Güllüpınar, B., Yanturali, S., Atilla, R., \& Musal, B. (2016). The comparison of the efficiency of traditional lectures to videosupported lectures within the training of the Emergency Medicine residents. Turkish journal of emergency medicine, 16(3), 107-111.

62. Seeley, C. L. (2017). Turning Teaching Upside Down. Educational

Leadership, 75(2), 32-36.

63. Senthamarai, S. (2018). Interactive teaching strategies. Journal of Applied and Advanced Research, 3(1), S36-S38.

64. Shah, I. P. (2019). comparison of small group discussion vs lecture method in teaching organon of medicine to under graduate homoeopathic

students. International Journal of Medical and Biomedical Studies, 3(8).

65. Shah, V., \& Jain, U. J. (2016). The effectiveness of integrated teaching over traditional teaching in third MBBS students. International Journal of medical science and Public Health, 5(7), 1430.

66. Shamsudin, M., Othman, M., Jahedi, M., \& Aralas, D. (2017). 
Enhancing English Learners'

Willingness to Communicate through Debate and Philosophy Inquiry Discussion. English Language Teaching, 10(8), 145152.

67. Sivarajah, R. T., Curci, N. E., Johnson, E. M., Lam, D. L., Lee, J. T., \& Richardson, M. L. (2019). A review of innovative teaching methods. Academic radiology, 26(1), 101-113.

68. Strubbe, L. E., Madsen, A. M., McKagan, S. B., \& Sayre, E. C. (2020). Beyond teaching methods: Highlighting physics faculty's strengths and agency. Physical Review Physics Education Research, 16(2), 020105.

69. Su, J. X., Cai, L. S., Chen, G., Dai, Y., Zhang, H. M., Gong, Z. L., \& Che, S. W. (2016). Exploration and Application of Discussion-based Teaching Method in Teaching of Medical Parasitology. Zhongguo ji sheng chong xue yu ji sheng chong bing za zhi= Chinese journal of parasitology \& parasitic diseases, 34(5), 473-475.

70. Sudarmika, P., Santyasa, I. W., \& Divayana, D. G. H. (2020). Comparison between Group Discussion Flipped Classroom and Lecture on Student Achievement and Student Characters. International Journal of Instruction, 13(3), 171-186.

71. Sun, G., \& Bin, S. (2018). Topic Interaction Model Based on Local Community Detection in MOOC Discussion Forums and its Teaching. Educational Sciences: Theory \& Practice, 18(6).

72. Tavoosy, Y., \& Jelveh, R. (2019). Language teaching strategies and techniques used to support students learning in a language other than their mother tongue. International Journal of Learning and Teaching, 11(2), 77-88.

73. Usarov, J. (2019). Using Teaching Methods for Development Student Competencies. International Journal of Progressive Sciences and Technologies, 15(1), 272-274.

74. Wang, Z., Akhter, S., \& Qureshi, A. H. (2020). Towards the Description of English Idioms for EFL Learners from Learning and Teaching Perspective. Revista Argentina de Clínica Psicológica, 29(3), 285.

75. Wang, X. (2016). Discussion on application of multimedia teaching in college English vocabulary teaching. Open Journal of Modern Linguistics, 6(3), 177-181.

76. Wei, L. I. U. (2014). On Issues of Discussion Teaching Method [J]. Journal of Changchun University, 24(02), 274-277.

77. Xhomara, N. (2020). Lecture and discussion methods as predictors of knowledge building by students Nazmi Xhomara. PSYCHOLOGY AND EDUCATION, 57(1), 18-24.

78. Yi, L., Ying, Z., \& Wijaya, T. T. (2019). The Trend of Mathematics Teaching Method Has Change from Fragments to Systematics. Jurnal Cendekia: Jurnal Pendidikan Matematika, 3(2), 471-480.

79. Yli-Panula, E., Jeronen, E., Lemmetty, P., \& Pauna, A. (2018). Teaching methods in biology promoting biodiversityeducation. Sustainabili ty, 10(10), 3812.

80. YOU, W. H., XI, D. G., FANG, L. X., \& DU, D. L. (2017). Discussion on Teaching Reforms of the 
Treatment and Disposal of Solid

Waste in Environmental Engineering. In Education Teaching Forum (No. 12, p. 40).

81. Yusupov, R. (2020). Modern informatics and its teaching methods. Архив Hayчных Публикаций JSPI, 1-10.

82. Zaidi, U., Hammad, L. F., Awad, S. S., Qasem, H. D., \& Al-Mahdi, N. A. (2017). Problem-based learning vs. traditional teaching methods: self-efficacy and academic performance among students of health and rehabilitation sciences college, PNU. Rehabilitation, 55, 547-551.

83. Zhao, B., \& Potter, D. D. (2016). Comparison of lecture-based learning vs discussion-based learning in undergraduate medical students. Journal of surgical education, 73(2), 250-257.

84. Zlotskaya, E. M. (2016). Traditional and modern teaching methods and information technologies in L2 teaching. In Человек, общество и государство в современном мире (pp. 352-357). 